\title{
The Effectiveness of Single-Dose Azithromycin Versus its Combination With Moxifloxacin Based on the Treatment of Chlamydial Cervicitis: A Study Protocol for a Randomized Clinical Trial
}

\author{
Farnaz Mohammadzadeh $^{1}$, Mahrokh Dolatian $^{2^{*} \mathbb{D}}$, Masoumeh Jorjani $^{3}$, Maryam Afrakhteh ${ }^{4}$, Hamid Alavi \\ Majd $^{5}$, Mostafa Qorbani ${ }^{6,7}$
}

\begin{abstract}
Objectives: Chlamydia trachomatis is globally reported as a pathogenic infection and is the most common bacterial cause of sexually transmitted infection (STI) in most countries. In addition, chlamydial cervicitis and the adverse complications of the first line treatment, namely, azithromycin failure are highly prevalent. Thus, the present study will be conducted to determine and compare the effects of single-dose azithromycin and its combination with moxifloxacin based on syndromic and etiologic methods in the diagnosis and treatment of chlamydial cervicitis.

Materials and Methods: This is a study protocol for a randomized clinical trial which will be performed at the Women's Clinics and Health Centers in Amol, Iran during 2018. Further, the study will evaluate women with cervicitis complaints, who have a proven case of chlamydial cervicitis through syndromic or etiologic diagnostic methods and meet the inclusion criteria for the study population. Discussion: To the best of our knowledge, this trial will be the first study to explore the effects of single-dose azithromycin in combination with moxifloxacin in the treatment of chlamydial cervicitis. The results of this trial will further reveal the diagnostic value of syndromic and etiologic methods in the diagnosis of chlamydial cervicitis.

Keywords: Trial protocol, Azithromycin, Moxifloxacin, Chlamydial cervicitis
\end{abstract}

\section{Introduction}

Chlamydia trachomatis is the most common bacterial cause of sexually transmitted infection (STI) in most countries. This intracellular Gram-negative bacterium can lead to cervicitis as well (1). According to the latest World Health Organization (WHO) reports, the prevalence of chlamydia has demonstrated a steady increase over the last two decades $(2,3)$ and over 100 million chlamydia infections are annually detected worldwide (4). In addition, the incidence of this disease is reported as 5\%$32.7 \%$ and 3\%-23.3\% among women and men in Iran (5).

Genital chlamydia infection is idiopathic in the majority of patients and its symptoms wide range from cervicitis to pelvic inflammatory disease (PID). Further, salpingitis, tubular ovarian abscess, and infertility are possible in frequent infections and ectopic pregnancy is regarded as an indirect complication of this infection. Furthermore, chlamydia infection increases the possibility of comorbidity with human immunodeficiency virus (6-10).

The treatment regimen for a subject treated for a disease or disorder associated with infection due to chlamydia include treatment with antibiotics, including but not limited to azithromycin, doxycycline, erythromycin base, levofloxacin, and/or ofloxacin, singly or in any combination (11-13). Azithromycin, as a first-line treatment, expresses its antibacterial activity primarily by reversible binding to the bacterial $50 \mathrm{~S}$ ribosomal subunits and blocking the progression of nascent proteins through their exit tunnel in bacterial protein biosynthesis (14). The side effects of azithromycin encompass digestive disorders in the short term and hearing loss in the long term (15). Moxifloxacin is a broad-spectrum antibiotic that is active against both Gram-positive and Gramnegative bacteria. It functions by inhibiting DNA gyrase, 
a type II topoisomerase, and topoisomerase IV, which are the necessary enzymes for separating bacterial DNA, thus inhibiting the cell replication (16). Moreover, diarrhea, dizziness, lightheadedness, headache, weakness, or trouble sleeping may occur by using moxifloxacin (17).

Several recent studies have also reported azithromycin treatment failure (18-24). In trying to reduce these failures, diagnostic advances are made in relation to chlamydial cervicitis. Among the diagnostic methods, the nucleic acid amplification test (NAAT) is the gold standard for the diagnosis of chlamydia (25-32). According to the latest WHO guidelines, comparative evidence for the effects of various treatment regimens are poor to moderate and thus further randomized, controlled, clinical trials are required to compare these treatments. This guideline strongly recommends the countries to update this universal guideline based on their standard national protocols, epidemiological status, and bacterial resistance data from their regions (33).

Many studies have evaluated the effect of azithromycin in the treatment of chlamydial cervicitis. However, studies on the use of the combination antibiotics, which reduce bacterial resistance and cover more strains of the pathogens, are involved in chlamydial cervicitis. Additionally, studies on comparing the diagnostic value of syndromic (only based on patient complaints and clinical examinations) and etiologic (both syndromic and laboratory diagnosis with NAAT) methods are scarce (34-36).

Therefore, this trial, to the best of our knowledge, is the first study to explore the effects of single-dose azithromycin in combination with moxifloxacin in the treatment of chlamydial cervicitis. In addition, the results from this trial investigate the diagnostic value of syndromic and etiologic diagnostic methods in the diagnosis of chlamydial cervicitis.

\section{Methods and Materials}

Study Design

This is a randomized, single-blind, clinical trial. The flow chart of the study protocol is presented in Figure 1. This study will be conducted at the Women's Clinics and Health Centers in Amol, located in the north of Iran.

\section{Sample Size}

The sample size was determined as 100 using the following formula:

$$
n \geq \frac{\left(z_{\alpha / 2}+z_{\beta}\right)^{2}}{\log ^{2}(O R)}\left(\frac{1}{\left(P_{1}\left(1-P_{1}\right)\right)}+\frac{1}{\left(P_{2}\left(1-P_{2}\right)\right)}\right)
$$

where the percentage failure estimate in the treatment groups, as well as the odds ratio, the test significance level, and the test power are considered as follows based on previous studies $(20,37)$ :

$$
\begin{aligned}
& P_{1}=0.15 \\
& P_{2}=0.03 \\
& \text { OR }=6 \\
& \alpha=0.05 \Rightarrow z_{\alpha / 2}=1.96 \\
& \beta=0.20 \Rightarrow z_{\beta}=0.85 \\
& \text { power }=0.80
\end{aligned}
$$

Further, the sample size was raised to 120 by taking account of potential attrition of $20 \%$.

\section{Study Population}

The population of the study contain all the women complaining of cervicitis, who visit the clinics and health centers in Amol, Mazandaran in the north part of Iran.

\section{Inclusion and Exclusion Criteria}

The inclusion criteria include being within the age range of 18-35 years, being a married and sexually active woman with only one sexual partner over the last two months. In addition, another criterion is having chlamydial cervicitis based on a series of symptoms found in the medical history and examination (in the syndromic diagnosis group) or a clinical diagnosis of chlamydial cervicitis confirmed by the real-time polymerase chain reaction (PCR) in the etiologic PCR diagnosis group. The other inclusion criteria are as follows:

- The rejection of gonococcal cervicitis by gram staining;

- The lack of the use of vaginal medications or antibiotics or immunosuppressive medications over the last 14 days;

- The rejection of complicated cervicitis (by cytology) in the Pap smear fluid or a PID comorbidity;

- The lack of pregnancy or pregnancy intention for the next three months;

- The lack of breastfeeding;

- The lack of using an intrauterine device;

- No history of allergy to compounds containing quinolones or macrolides;

- No concurrent use of medications interacting with azithromycin or moxifloxacin (e.g., antipsychotics, tricyclic antidepressants, antiarrhythmics, nonsteroidal anti-inflammatory drugs, anticoagulants, and the like).

The exclusion criteria will encompass the necessity for using antibiotics during the treatment and medications that interact with macrolides and fluoroquinolones (e.g., digoxin, cyclosporine, and the like), as well as pregnancy during the treatment, the intolerance of the medications in any form due to the side-effects, and the discontinuation of the medications for at least 48 hours.

\section{Randomization}

After the coding stage, the subjects will be randomly assigned to four treatment groups using Random Allocation Software as follows: 


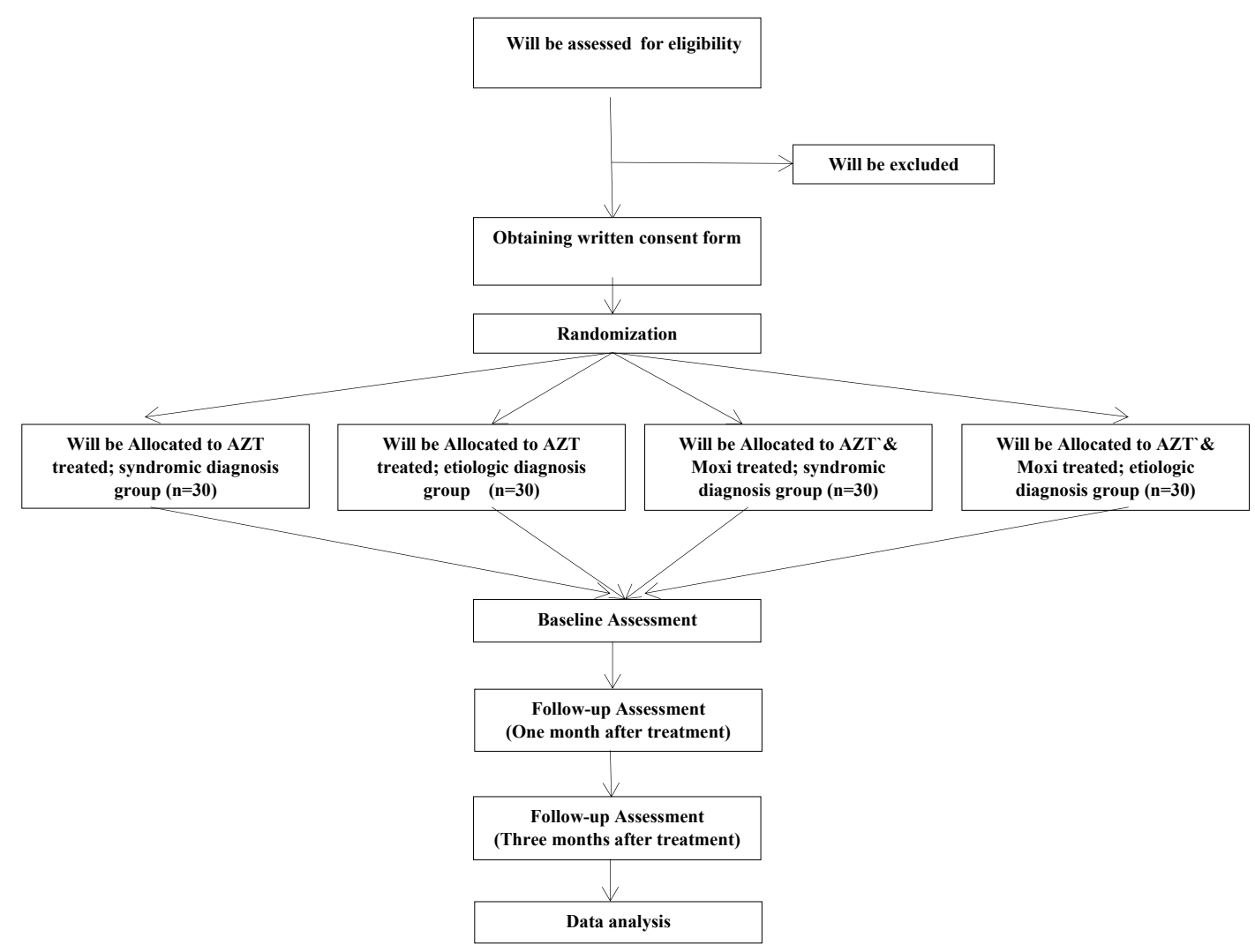

Figure 1. Subjects' Flow Diagram. Note. AZT: Azithromycin; Moxi: Moxifloxacin.

- The oral azithromycin and syndromic diagnosis group;

- The oral azithromycin and real-time (RT) PCR etiologic diagnosis group;

- The combination treatment with oral azithromycin and moxifloxacin and syndromic diagnosis group;

- The combination treatment with oral azithromycin and moxifloxacin and RT-PCR etiologic diagnosis group.

The groups will be followed-up one month and three months after the treatment.

\section{Intervention}

In general, $1000 \mathrm{mg}$ azithromycin (two tabs $500 \mathrm{mg}$ ) are orally administered as a single dose, which is manufactured by Chemidaru Pharmaceutical Company (GTIN: 06260154636041; IRC: 2366570648796929). One $400 \mathrm{mg}$ moxifloxacin tablet per day is administered for seven days, which is manufactured by Darou-Pakhsh Pharmaceutical Company (GTIN: 06260132430517; IRC: 2493594828199352). In the azithromycin and moxifloxacin combination treatment, two 500-mg azithromycin tablets are orally administered as a single dose on the first day, and then treatment continues with the administration of one $400 \mathrm{mg}$ moxifloxacin tablet per day from the second to the eighth day (for seven days). The same dose of the allocated medication is also administered to the subjects' spouses in each of the four groups.
In the syndromic diagnostic method, the evaluated cervicitis complaints will include mucopurulent vaginal discharge, vulvar and vaginal irritation and burning, lower abdominal pain, and painful intercourse. In the etiologic diagnostic method, RT-PCR is used to confirm the diagnosis following these assessments, and then bacterial DNA is extracted using the commercial kit (5-primeGermany) according to the instructions. Furthermore, the patients' infection with chlamydia trachomatis is then assessed by PCR and the Russian commercial interlaboratory kit according to the instructions. The subjects are asked to refrain from having sex during the treatment and use condoms in the follow-up period. They are also required to avoid having intercourse or using vaginal douches and spermicides or other vaginal medications and antibiotics while taking the medications.

\section{Adherence}

The researcher contacts the subjects on their phone every week to assess their complaints and the possible questions and to ensure their proper use of medications during the treatment and in the one-month and three-month posttreatment follow-ups.

\section{Patient Safety}

Each subject is given written instructions for using the medications and a note containing some hygienic tips. In addition to the weekly phone calls, a self-reporting form 
is provided to the subjects to complete every night and bring to the follow-ups so as to evaluate their correct use of the medications and listen to their possible complaints (vaginal and vulvar burning and irritation, along with discharge and abdominal pain). The subjects are requested to contact the researcher or visit the study setting in case of any problems or the medicinal side-effects or if they have any questions. In addition, the researcher contacts the subjects with prior arrangements in order to ensure their correct use of the medications and remind them of their visit schedule.

\section{Results}

Primary Outcomes

The primary outcomes of this clinical trial include changing the patients' complaints, clinical examination findings, and laboratory results in all treatments groups, as well as disease recurrence during the one-month and three-month follow-ups.

The secondary outcomes of this clinical trial contain the effect of the treatment and diagnostic methods on the experimental groups and the recurrence of the disease during the one-month and three-month follow-ups.

\section{Procedures}

First, women aged 18-35 years, who complaint of cervicitis (i.e., abnormal yellow or green vaginal discharge, vaginal and vulvar burning and irritation, lower abdominal pain, and painful intercourse), will complete a preliminary questionnaire. Then, eligible patients, who meet the inclusion criteria (i.e., having no sexual intercourse in certain stages of the study, the compulsory use of condoms in some other stages, no use of vaginal douches or other vaginal medications, and no use of antibiotics) and accept the study conditions will complete the consent forms and undergo an examination following taking their samples.

After the coding stage, these subjects will be randomly divided into four experimental groups using random allocation software as follows:

- Group 1: Oral azithromycin and syndromic diagnosis (only based on patient complaints and clinical examinations);

- Group 2: Oral azithromycin and etiologic diagnosis (both syndromic and laboratory diagnosis with RTPCR);

- Group 3: Combination treatment with oral azithromycin and moxifloxacin and syndromic diagnosis;

- Group 4: Combination treatment with oral azithromycin and moxifloxacin and etiologic diagnosis.

Then, the researcher will evaluate the subjects for the signs of genital lesions, ulcers, and warts in the lithotomy position. Genital ulcers are generally caused by herpes simplex and genital warts are mostly caused by human papillomavirus. The subjects with herpetic lesions or genital warts are excluded from the study. Then, by inserting a sterile speculum (without any lubricants), the vagina and cervix will be examined by the researcher for symptoms such as erosion, inflammation, edema, erythema and discharge color, odor, as well as consistency and amount, and the observed cases will be recorded accordingly. In the speculum examination, patients with symptoms of PID (i.e., the triad: pelvic pain, sensitivity to the mobility of cervix, and adnexal sensitivity) or abnormal cervix will be excluded from the study. The patient's cervical mucosa is swollen and brittle and purulent mucopurulent mucosal discharge is observed in chlamydial infections. If yellow or green purulent endocervical discharge (mucopus) is detected, the cervical mucus will then be extracted by inserting a small cotton swab into the endocervical tract and viewed against a white or black background to detect whether it is green or yellow.

Sensitivity to touch will also be assessed during cervical and urethral tract movements, followed by examining cervical erythema, along with fragility and central erosion with a hypertrophic appearance. The ectopic area (glandular epithelium) can fragility be evaluated by having a cotton swab or spatula touching the ectropion region. Accordingly, the chlamydial infection is diagnosed for the subjects in the "syndromic diagnosis" group if the aforementioned cases are positive. Several actions are taken to reject gonorrheal cervicitis and take the samples from the cervix. The excess endocervical mucus is first cleaned up by a piece of cotton, and a dacron swab is inserted $(2 \mathrm{~cm})$ into the endocervical canal and passed through the squamous-cylindrical junction (so that its tip is not invisible). Then, it is gently turned 360 degrees clockwise or anticlockwise toward one side, left for 15 seconds, and finally, removed (epithelial cell sampling is necessary even with canal discharge since chlamydia trachomatis is an intracellular organism and discharge alone is insufficient). The contact between the swab and the exocervical cells or vagina should be avoided when removing the swab. The researcher will place the mucopus on a slide, and a neardefinitive diagnosis of gonorrheal endocervicitis made when observing intracellular Gram-negative diplococci (inside the neutrophils) using the Gram-staining. Then, the patient is excluded from the study and referred to a gynecologist. A cytobrush is also used to perform the liquid-based Pap smear and the samples will be transferred to the laboratory. The researcher will then request to receive the results within 24 hours as well. The sample is excluded from the study and referred to a gynecologist if there is a report on any epithelial cell abnormality.

The researcher examines the Amsel criteria if the sample is diagnosed with a clear bacterial vaginosis infection in the clinical examination. For this purpose, the vaginal discharge $\mathrm{pH}$ is measured after one minute of the contact between a $\mathrm{pH}$ testing tape (Merck, Germany; range: 0 to 14 ) and the vaginal wall. The $\mathrm{pH}$ should not be measured utilizing the samples taken from the posterior 
vaginal fornix since its measurement may be higher in this region due to the presence of the cervical mucus. Next, the discharge from the side walls and the vaginal posterior fornix are collected using two sterile swabs. One to two drops of normal saline are added to the first sample after it is placed on the slide, and the sample is then assessed under the microscope (with a magnification of 400x) in terms of the presence of the key cells. In addition, the discharges collected by the second swab are placed on a clean glass slide and then evaluated in terms of amine odor (the Whiff test) after the addition of a drop of potassium hydroxide $10 \%$. The bacterial vaginosis is diagnosed based on the clinical Amsel criteria and if three out of the following four conditions are met:

- Having a $\mathrm{pH} \geq 4.5$;

- Observing the key cells in the first slide;

- Representing a positive amine test in the second slide;

- Having white-gray homogeneous discharge.

By diagnosing the bacterial vaginosis (based on the Amsel criteria), the patient's already-assigned treatment is supplemented with $500-\mathrm{mg}$ oral metronidazole tablets taken one every 12 hours for seven days.

In the samples that are in the RT-PCR etiologic diagnostic group, the same sample of liquid-based Pap smear will simultaneously be used to conduct a NAAT for assessing the chlamydia while doing the above steps in the syndromic diagnostic group. The endocervical samples are taken by inserting a cytobrush (for about $2 \mathrm{~cm}$ ) into the endocervical canal and then turning it for at least 30 seconds in the opposite direction to the canal wall. The endocervical discharge is placed in a preserver $(2 \mathrm{~mL}$ of the sterile transportation medium containing alcoholbased preservative liquid as a fixator), which can be kept for weeks at room temperature. This liquid is immediately transferred to the laboratory, where the blood and inflammatory exudate are removed from the prepared cell suspension for samples homogenization.

The bacterial DNA is extracted using a commercial kit (5-prime, Germany) according to the instructions. In brief, $900 \mu \mathrm{L}$ of vaginal discharge and $400 \mu \mathrm{L}$ of the buffer solution are added to the extraction column and centrifuged at $10000 \mathrm{~g}$ for 10 seconds. The column is transferred to a new micro-tube and rinsed again under the aforementioned conditions, followed by the completion of the DNA extraction process from the column by adding $70 \mu \mathrm{L}$ of the extraction solution to the column and centrifuging it. After the extraction of DNA from the samples based on the 5-prime kit instructions, the infection of the samples with chlamydia trachomatis is evaluated utilizing the PCR and the Russian interlaboratory kit (according to the kit instructions). The PCR test results are reported to the researcher within 24 hours. Negative samples are excluded from the study and referred to a gynecologist. Then, the researcher contacts the patients with positive samples as per the PCR and invite them to visit the study setting to receive their medications based on the initial random group allocation. After the diagnosis of infection with chlamydia, measures are taken depending on the group to which the subject belongs, and the routine treatment group is given two 500-mg oral azithromycin tablets (as a single dose). In addition, the combination treatment group receives two 500-mg oral azithromycin tablets (as a single dose) for day one and then $400 \mathrm{mg}$ oral moxifloxacin tablets once per day for seven days (for the second to eighth days).

The same dose of the assigned medication is administered to the subjects' spouses in all four groups. Each person also receives written medication use instructions and a note containing the hygienic tips. Further, self-reporting form is given to the subjects to assess their correct use of the medications and to write down their potential complaints (e.g., burning, discharge, and abdominal pain) every night and bring them to the follow-ups. The subjects are asked to contact the researcher or visit the study setting in the case of problems, medication side-effects, or any questions they may have in this regard. Considering the prior arrangements, the researcher contacts the subjects to assure them of their correct medication consumption and remind them of their visit schedule. Moreover, the subjects are advised to start their treatment within at most the next 24 hours and record possible medication side-effects. They are also instructed to avoid intercourse and the use of vaginal douche and spermicides or any other vaginal medications or antibiotics while taking the medications.

The subjects will again visit a month after starting their treatment to submit their self-reporting forms and be re-evaluated in terms of clinical and laboratory criteria according to their initial random group allocations. The obtained results are recorded in the observation form, followed by noting the medication side-effects. Those with no clinical or laboratory recovery are removed from the research and referred to a gynecologist. Next, the recovered subjects are contacted with three months after the treatment and asked to visit the study setting to determine re-infection with chlamydia during the followup period. They are then re-evaluated for the signs of chlamydia infection based on the clinical and laboratory criteria and according to their initial random group allocations and the results are noted in the observation form. Finally, in all 120 subjects (30 subjects per group), the results of the statistical tests will be assessed regarding the comparison between the four groups, as well as the results related to the diagnostic value of the two methods, the recovery achieved with the two medications, and their side-effects.

\section{Statistical Analysis}

All the statistical analyses are performed by the SPSS software, version 22 (SPSS Inc., Chicago, USA). Continuous and categorical variables are expressed as mean (SD) and number (percentage), respectively. The normal distribution of continuous variables are 
assessed using the Kolmogorov-Smirnov test, followed by utilizing the chi-square and Kruskal-Wallis statistical tests to compare the demographic data of patients in different treatment groups. The missing values will be imputed by applying the regression method. Additionally, the results of fitting the logistic regression model are applied to compare the recovery of patients' complaints, the improvement of clinical observations, as well as the treatment and drug complications after the intervention in four different therapeutic groups. Group 4 (Combination treatment with oral azithromycin and moxifloxacin and etiologic diagnosis) is the reference group and the other three groups are compared with this group and with each other. The $P<0.05$ is considered as the level of statistical significance.

\section{Ethical Considerations}

The present clinical trial is conducted after the approval of the Medical Ethics Committee of Shahid Beheshti University of Medical Sciences and obtaining informed consent from the subjects. They are ensured of their right to participate in (if meeting the eligibility criteria) or withdraw from the study at any stage and visit or contact in the case of any problems or questions. The women are also ensured of the confidentiality of their data, and no expenses are incurred by them. Proper medication storage conditions should be observed and the medications should be discontinued at any stage in the case of demonstrating the adverse effects.

\section{Discussion}

Chlamydia trachomatis is regarded as one of the most common sexually-transmitted pathogens in humans. Chlamydia infection can affect several organs including the penis, vagina, cervix, urethra, and anus and thus can cause serious and occasionally permanent damage to the reproductive system. Azithromycin is a group of macrolides that is effective on urethritis or non-gonococcal cervicitis caused by chlamydia trachomatis in addition to pelvic inflammatory infections due to chlamydia trachomatis and mycoplasma hominis. In addition, moxifloxacin is an antibiotic of the fourth generation of fluoroquinolones, which affects a wide range of Gram-negative and Grampositive bacteria in the urogenital region. This antibiotic is an effective therapeutic option for patients with aerobic vaginitis as well $(38,39)$. The disease complaints in women include dysuria, vaginal discharge, irregular uterine bleeding, painful intercourse, and vague abdominal pain. Further, patients may complain of symptoms such as itching, pain, and urinary tract discharge or the symptoms of rectal involvement when experiencing anal intercourse. In frequent infections, salpingitis, tubular ovarian abscess, and infertility are also possible, and ectopic pregnancy is an indirect complication of this infection. The tissue damage following chlamydia trachomatis is noteworthy because it happens due to late diagnosis and treatment
(6-10). According to the World Health Organization (WHO) guidelines, only treatment regimens that have more than $95 \%$ efficacy are recommended for STIs (40). Although the present findings confirm the effect of azithromycin in the treatment of urogenital chlamydia, there are controversial reports on the rate difference of azithromycin failure with alternative treatments or combination treatments with doxycycline. For example, Kissinger et al showed that the treatment failure of 1-g single-dose oral azithromycin was between $6.2 \%$ and $12.8 \%$ in men with chlamydial urethritis who had sexual relationships with women with chlamydial cervicitis, which exceeds the WHO target range (33). Similarly, Kong et al compared the treatment effects of 1-g single-dose oral azithromycin and 1-g single-dose oral azithromycin plus 100-mg doxycycline twice daily for seven days in men with anal chlamydia and reported the failure rates of $12.8 \%$ and $0 \%$ in the 2 groups, respectively (41). Kong et al also conducted a meta-analysis to determine and compare the effects of azithromycin and doxycycline in the treatment of genital chlamydial infection and found the failure rates of $4 \%$ and $3 \%$, respectively (42). In another study, Geisler et al observed the failure rates of $15.2 \%$ and 7.9\% for 25-mg single dose of rifalazil and 1-g single-dose of azithromycin in women with genital chlamydia (43). Likewise, Geisler et al conducted a study on men and women with urogenital chlamydia who were randomly assigned to receive 1-g single-dose azithromycin or 100 mg doxycycline twice daily for seven days, and reported the effect of azithromycin as $97 \%$ and doxycycline as $100 \%$ (44). In a prospective cohort study, Dukers-Muijrers et al evaluated the rate of recovery from anorectal and cervicovaginal chlamydia following treatment with $1-\mathrm{g}$ single-dose of oral azithromycin and indicated that the treatment failure rate was equal to $15-27 \%$ after three to eight weeks of post-treatment follow-up (45). Moreover, Schwebke et al investigated the effects of 1-g single-dose azithromycin and $100 \mathrm{mg}$ doxycycline twice daily for seven days, as well as the effect of adding $2 \mathrm{~g}$ of tinidazole to these treatment regimens. They showed that the addition of tinidazole failed to improve the recovery rate, but effectively eradicated chlamydia. Based on their results, no significant differences were found between the treatment groups in terms of the clinical recovery rate, but the effect of doxycycline was significantly higher than that of azithromycin such that treatment failure was reported as $22.6 \%$ and $5.2 \%$ in azithromycin and doxycycline groups, respectively (46). Previous research demonstrated that patients with the symptoms of chlamydial infection have high organic loads, which could contribute to their failure of treatment with azithromycin (47).

Recent studies have revealed that inadequate antibiotic coverage against the associated bacteria in chlamydial cervicitis is one of the most prevalent causes of the failure of the treatment. Additionally, some studies indicated that mycoplasma genitalium is one of the most common 
bacteria associated with chlamydia trachomatis, and moxifloxacin is one of the most effective drugs for the treatment of mycoplasma genitalium $(21-24,48)$. Based on the reports of some studies, mycoplasma genitalium treatment failed with macrolides such as azithromycin. Several recent studies have also reported the prevalence of mutations related to the resistance of strains associated with chlamydia trachomatis against azithromycin and moxifloxacin as $6.6 \%$ and $41.4 \%$. Resistance to treatment with a combination of azithromycin and moxifloxacin was found to be $2.7 \%(1.1 \%-4.2 \%)$ as well $(49,50)$. Nonetheless, an in-vitro study represented the potential effects of moxifloxacin on chlamydial species (51).

Given the high percentage of single-dose-azithromycin treatment failure in chlamydial infections and the increasing concerns about the serious complications caused by the low-moderate effect of this medication, the present study was designed as the first randomized, controlled, clinical trial to compare the effect of singledose azithromycin and azithromycin plus moxifloxacin and assess the clinical and laboratory recovery rates, as well as the complications related to the use of each of these treatment regimens.

The limitations of this study include the noncooperation of some of the patients, who will be replaced by other patients. In addition, subjects unpunctual visits in spite of their consent for participation is another study limitation that should be rectified by the researcher's attempts to explain the necessity of attending the followup sessions and making reminder phone calls to encourage the subjects. Refraining from sexual intercourse during the first follow-up period and using condoms in the second are the other limitations, and the researcher shall emphasize their importance in face-to-face meetings and phone calls.

Therefore, future studies can include clinical trials on the therapeutic effects of different doses of azithromycin or the combined therapy with antibiotics affecting the common bacteria associated with chlamydia trachomatis.

\section{Conflict of Interests}

Authors declare that they have no conflict of interests.

\section{Ethical Issues}

The study protocol was approved by the Medical Ethics Committee of Shahid Beheshti University of Medical Sciences (IR.SBMU.RETECH.REC.1397.309) and registered at the website of the Iranian Registry of Clinical Trials (IRC20100130003226N16). The informed consent forms will be signed by the subjects at the beginning of the study.

\section{Financial Support}

This research is supported by Shahid Beheshti University of Medical Sciences, Tehran, Iran.

\section{References}

1. Gwyn SE, Xiang L, Kandel RP, Dean D, Gambhir M, Martin DL. Prevalence of Chlamydia trachomatis-Specific Antibodies before and after Mass Drug Administration for Trachoma in Community-Wide Surveys of Four Communities in Nepal. Am J Trop Med Hyg. 2018;98(1):216-220. doi:10.4269/ajtmh.17-0102

2. Zambrano AI, Sharma S, Crowley K, et al. The World Health Organization Recommendations for Trachoma Surveillance, Experience in Nepal and Added Benefit of Testing for Antibodies to Chlamydia trachomatis pgp3 Protein: NESTS Study. PLoS Negl Trop Dis. 2016;10(9):e0005003. doi:10.1371/journal.pntd.0005003

3. Heijne $\mathrm{M}$, van der Goot JA, Fijten $\mathrm{H}$, et al. A cross sectional study on Dutch layer farms to investigate the prevalence and potential risk factors for different Chlamydia species. PLoS One. 2018;13(1):e0190774. doi:10.1371/journal. pone. 0190774

4. Ige O, Ige S, Olayinka A. Prevalence of Chlamydia Trachomatis infection among women of reproductive age group in a tertiary hospital in Northern Nigeria. Ann Trop Pathol. 2018;9(1):17-21. doi:10.4103/atp.atp_35_17

5. Ahmadi MH, Mirsalehian A, Bahador A. Prevalence of genital Chlamydia trachomatis in Iran: a systematic review and meta-analysis. Pathog Glob Health. 2015;109(6):290299. doi: $10.1179 / 2047773215 y .0000000033$

6. Giffard PM, Lilliebridge RA, Wilson J, et al. Contaminated fingers: a potential cause of Chlamydia trachomatis-positive urine specimens. Sex Transm Infect. 2018;94(1):32-36. doi:10.1136/sextrans-2016-053081

7. Schuchardt L, Rupp J. Chlamydia trachomatis as the Cause of Infectious Infertility: Acute, Repetitive or Persistent Long-Term Infection? Curr Top Microbiol Immunol. 2018;412:159-182. doi:10.1007/82_2016_15

8. Reekie J, Donovan B, Guy R, et al. Risk of Pelvic Inflammatory Disease in Relation to Chlamydia and Gonorrhea Testing, Repeat Testing, and Positivity: A Population-Based Cohort Study. Clin Infect Dis. 2018;66(3):437-443. doi:10.1093/cid/ cix769

9. Olson KM, Tang J, Brown L, Press CG, Geisler WM. HLA$\mathrm{DQB1}^{\star} 06$ is a risk marker for chlamydia reinfection in African American women. Genes Immun. 2019;20(1):6973. doi:10.1038/s41435-018-0014-3

10. Lohi K, Kumar C, Fonseca M, Baveja S, Panchal S. Study of Chlamydia trachomatis Infection in Infertile Females at a Tertiary Care Hospital in Mumbai, India. Int J Curr Microbiol Appl Sci. 2018;7(3):1985-2000. doi:10.20546/ ijcmas.2018.703.234

11. Tsang AW, Furdui CM. Method of treatment of chlamydial infections with selected EGFR inhibitors. Google Patents; 2018.

12. Peuchant O, Lhomme E, Kret M, et al. Randomized, open-label, multicenter study of azithromycin compared with doxycycline for treating anorectal Chlamydia trachomatis infection concomitant to a vaginal infection (CHLAZIDOXY study). Medicine (Baltimore). 2019;98(7):e14572. doi:10.1097/md.0000000000014572

13. Wilson N, Goodhew B, Mkocha H, et al. Evaluation of a Single Dose of Azithromycin for Trachoma in LowPrevalence Communities. Ophthalmic Epidemiol. 2019;26(1):1-6. doi:10.1080/09286586.2017.1293693

14. Jelić D, Antolović R. From Erythromycin to Azithromycin and New Potential Ribosome-Binding 
Antimicrobials. Antibiotics (Basel). 2016;5(3). doi:10.3390/ antibiotics5030029

15. Astale T, Sata E, Zerihun M, et al. Self-Reported Side Effects following Mass Administration of Azithromycin to Eliminate Trachoma in Amhara, Ethiopia: Results from a Region-Wide Population-Based Survey. Am J Trop Med Hyg. 2019;100(3):696-699. doi:10.4269/ajtmh.18-0781

16. Qiu Z, Yuan H, Li N, et al. Bidirectional effects of moxifloxacin on the proinflammatory response in lipopolysaccharidestimulated mouse peritoneal macrophages. Mol Med Rep. 2018;18(6):5399-5408. doi:10.3892/mmr.2018.9569

17. Kwon S, Lee DH, Kang JB, et al. [The Efficacy of Bismuthcontaining Quadruple Therapy after Moxifloxacinbased Sequential Therapy Failure in Helicobacter pylori Eradication]. Korean J Gastroenterol. 2018;71(4):196-203. doi:10.4166/kjg.2018.71.4.196

18. Lusk MJ, Garden FL, Cumming RG, Rawlinson WD, Naing ZW, Konecny P. Cervicitis: a prospective observational study of empiric azithromycin treatment in women with cervicitis and non-specific cervicitis. Int J STD AIDS. 2017;28(2):120-126. doi:10.1177/0956462416628319

19. Couldwell DL, Lewis DA. Mycoplasma genitalium infection: current treatment options, therapeutic failure, and resistance-associated mutations. Infect Drug Resist. 2015;8:147-161. doi:10.2147/idr.s48813

20. Kissinger PJ, White S, Manhart LE, et al. Azithromycin Treatment Failure for Chlamydia trachomatis Among Heterosexual Men With Nongonococcal Urethritis. Sex Transm Dis. 2016;43(10):599-602. doi:10.1097/ olq.0000000000000489

21. Upton A, Bissessor L, Lowe P, Wang X, McAuliffe G. Diagnosis of Chlamydia trachomatis, Neisseria gonorrhoeae, Trichomonas vaginalis and Mycoplasma genitalium: an observational study of testing patterns, prevalence and co-infection rates in northern New Zealand. Sex Health. 2018;15(3):232-237. doi:10.1071/sh17110

22. Tamarelle J, Thiébaut ACM, de Barbeyrac B, Bébéar C, Ravel J, Delarocque-Astagneau E. The vaginal microbiota and its association with human papillomavirus, Chlamydia trachomatis, Neisseria gonorrhoeae and Mycoplasma genitalium infections: a systematic review and meta-analysis. Clin Microbiol Infect. 2019;25(1):35-47. doi:10.1016/j.cmi.2018.04.019

23. Taylor BD, Zheng X, O'Connell CM, Wiesenfeld HC, Hillier SL, Darville T. Risk factors for Mycoplasma genitalium endometritis and incident infection: a secondary data analysis of the T cell Response Against Chlamydia (TRAC) Study. Sex Transm Infect. 2018;94(6):414-420. doi:10.1136/ sextrans-2017-053376

24. Panos G. Prevalence studies of M. genitalium and other sexually transmitted pathogens in high risk individuals indicate the need for comprehensive investigation of STIs for accurate diagnosis and effective treatment. Germs. 2018;8(1):8-11. doi:10.18683/germs.2018.1127

25. Chambers LC, Khosropour CM, Katz DA, Dombrowski JC, Manhart LE, Golden MR. Racial/Ethnic Disparities in the Lifetime Risk of Chlamydia trachomatis Diagnosis and Adverse Reproductive Health Outcomes Among Women in King County, Washington. Clin Infect Dis. 2018;67(4):593599. doi:10.1093/cid/ciy099

26. Davies B, Turner KME, Benfield T, et al. Pelvic inflammatory disease risk following negative results from chlamydia nucleic acid amplification tests (NAATs) versus nonNAATs in Denmark: A retrospective cohort. PLoS Med. 2018;15(1):e1002483. doi:10.1371/journal.pmed.1002483

27. Migchelsen S, Wills G, Horner P, et al. P3.213 A tool for evaluating the impact of the national chlamydia screening programme in england: c. trachomatis antibody prevalence in young women in england (2007-2015). Sex Transm Infect. 2017;93(Suppl 2):A172. doi:10.1136/ sextrans-2017-053264.448

28. Cornelisse VJ, Chow EP, Huffam S, et al. Increased Detection of Pharyngeal and Rectal Gonorrhea in Men Who Have Sex With Men After Transition From Culture To Nucleic Acid Amplification Testing. Sex Transm Dis. 2017;44(2):114-117. doi:10.1097/olq.0000000000000553

29. Balla E, Donders GGG, Petrovay F, Urbán E. Seroprevalence of anti-Chlamydia trachomatis IgM in neonatal respiratory tract infections in Hungary. J Med Microbiol. 2017. doi:10.1099/jmm.0.000557

30. Harding-Esch EM, Cousins EC, Chow SC, et al. A 30-Min Nucleic Acid Amplification Point-of-Care Test for Genital Chlamydia trachomatis Infection in Women: A Prospective, Multi-center Study of Diagnostic Accuracy. EBioMedicine. 2018;28:120-127. doi:10.1016/j.ebiom.2017.12.029

31. Hay P. Vaginal discharge. Medicine. 2018;46(6):319-324. doi:10.1016/j.mpmed.2018.03.006

32. Thielemans E, Wyndham-Thomas C, Henrard S, et al. Screening for Chlamydia trachomatis and Neisseria gonorrhoeae Infections in Men Who Have Sex With Men: Diagnostic Accuracy of Nucleic Acid Amplification Test on Pooled Urine, Anorectal, and Pharyngeal Specimens. Sex Transm Dis. 2018;45(3):195-198. doi:10.1097/ olq.0000000000000722

33. World Health Organization (WHO). WHO guidelines for the treatment of Chlamydia trachomatis. WHO; 2016.

34. Hadad R, Golparian D, Lagos AC, et al. Macrolide and fluoroquinolone resistance in Mycoplasma genitalium in two Swedish counties, 2011-2015. APMIS. 2018;126(2):123127. doi:10.1111/apm.12792

35. Singh MM, Devi R. Time to rethink the syndromic approach to sexually transmitted infections. BMJ. 2017;358:j3789. doi:10.1136/bmj.j3789

36. Barry MS, Ba Diallo A, Diadhiou M, et al. Accuracy of syndromic management in targeting vaginal and cervical infections among symptomatic women of reproductive age attending primary care clinics in Dakar, Senegal. Trop Med Int Health. 2018;23(5):541-548. doi:10.1111/tmi.13046

37. Hocking J, Vodstrcil L, Huston W, et al. O15.4 Azithromycin treatment failure in women infected with genital chlamydia infection. Sex Transm Infect. 2015;91(Suppl 2):A59. doi:10.1136/sextrans-2015-052270.162

38. 38 Wang C, Han C, Geng N, et al. Efficacy of oral moxifloxacin for aerobic vaginitis. Eur J Clin Microbiol Infect Dis. 2016;35(1):95-101. doi:10.1007/s10096-0152513-8

39. Marks M, Bottomley C, Tome $\mathrm{H}$, et al. Mass drug administration of azithromycin for trachoma reduces the prevalence of genital Chlamydia trachomatis infection in the Solomon Islands. Sex Transm Infect. 2016;92(4):261265. doi:10.1136/sextrans-2015-052439

40. Workowski KA, Bolan GA. Sexually transmitted diseases treatment guidelines, 2015. MMWR Recomm Rep. 2015;64(RR-03):1-137.

41. Kong FY, Tabrizi SN, Fairley CK, et al. Higher organism 
load associated with failure of azithromycin to treat rectal chlamydia. Epidemiol Infect. 2016;144(12):2587-2596. doi:10.1017/s0950268816000996

42. Kong FY, Tabrizi SN, Law M, et al. Azithromycin versus doxycycline for the treatment of genital chlamydia infection: a meta-analysis of randomized controlled trials. Clin Infect Dis. 2014;59(2):193-205. doi:10.1093/cid/ciu220

43. Geisler WM, Pascual ML, Mathew J, et al. Randomized, double-blind, multicenter safety and efficacy study of rifalazil compared with azithromycin for treatment of uncomplicated genital Chlamydia trachomatis infection in women. Antimicrob Agents Chemother. 2014;58(7):40144019. doi:10.1128/aac.02521-14

44. Geisler WM, Uniyal A, Lee JY, et al. Azithromycin versus Doxycycline for Urogenital Chlamydia trachomatis Infection. N Engl J Med. 2015;373(26):2512-2521. doi:10.1056/NEJMoa1502599

45. Dukers-Muijrers NH, Speksnijder AG, Morre SA, et al. Detection of anorectal and cervicovaginal Chlamydia trachomatis infections following azithromycin treatment: prospective cohort study with multiple time-sequential measures of rRNA, DNA, quantitative load and symptoms. PLoS One. 2013;8(11):e81236. doi:10.1371/journal. pone.0081236

46. Schwebke JR, Rompalo A, Taylor S, et al. Re-evaluating the treatment of nongonococcal urethritis: emphasizing emerging pathogens--a randomized clinical trial. Clin Infect Dis. 2011;52(2):163-170. doi:10.1093/cid/ciq074

47. 47 Michel CEC, Sonnex C, Carne CA, et al. Chlamydia trachomatis load at matched anatomic sites: implications for screening strategies. J Clin Microbiol. 2007;45(5):13951402. doi:10.1128/jcm.00100-07

48. 48. Li Y, Le WJ, Li S, Cao YP, Su XH. Meta-analysis of the efficacy of moxifloxacin in treating Mycoplasma genitalium infection. Int J STD AIDS. 2017;28(11):1106-1114. doi: $10.1177 / 0956462416688562$

49. 49. Unemo M, Salado-Rasmussen $K$, Hansen $M$, et al. Clinical and analytical evaluation of the new Aptima Mycoplasma genitalium assay, with data on M. genitalium prevalence and antimicrobial resistance in M. genitalium in Denmark, Norway and Sweden in 2016. Clin Microbiol Infect. 2018;24(5):533-539. doi:10.1016/j.cmi.2017.09.006

50. 50. Mondeja BA, Couri J, Rodríguez NM, Blanco O, Fernández C, Jensen JS. Macrolide-resistant Mycoplasma genitalium infections in Cuban patients: an underestimated health problem. BMC Infect Dis. 2018;18(1):601. doi:10.1186/s12879-018-3523-9

51. 51. Miyashita N, Fukano H, Yoshida K, Niki Y, Matsushima T. In-vitro activity of moxifloxacin and other fluoroquinolones against Chlamydia species. J Infect Chemother. 2002;8(1):115-117. doi:10.1007/s101560200019

() 2019 The Author (s); This is an open-access article distributed under the terms of the Creative Commons Attribution License (http://creativecommons.org/licenses/by/4.0), which permits unrestricted use, distribution, and reproduction in any medium, provided the original work is properly cited. 